\title{
Mucosite oral em crianças com câncer: dificuldades de avaliação e de terapia efetiva
}

\author{
Oral mucositis in children with cancer: difficulties of evaluation and effective therapy \\ Mucositis oral en niños con cáncer: dificultades en la evaluación y terapia eficaz
}

Recebido: 30/08/2021 | Revisado: 02/09/2021 | Aceito: 03/09/2021 | Publicado: 05/09/2021

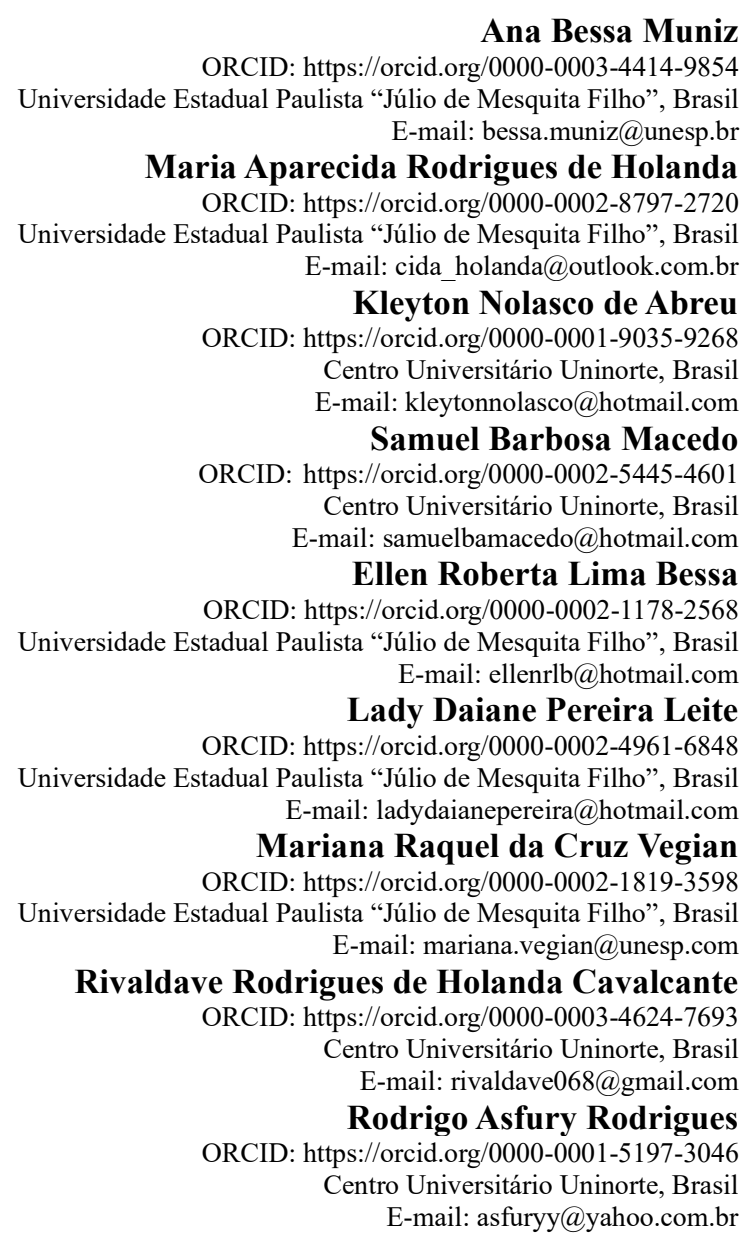

\section{Resumo}

O câncer é um problema de saúde pública no mundo e, em crianças, a incidência de aumento é de cerca de $1 \%$ ao ano. Apesar da eficácia, a terapia antineoplásica provoca efeitos de significativa toxicidade, muitas vezes causando a hospitalização do paciente, dificuldades de continuação da oncoterapia ou não readequação do tratamento para a não progressão da doença. A mucosite oral é um dos efeitos deletérios mais comuns; causa dor, afeta a fala e a nutrição e torna o paciente suscetível à septicemia. Em crianças, a frequência da mucosite oral situa-se em torno de $65 \%$. Suas manifestações são avaliadas por meio de escalas diferentes e tratadas com protocolos distintos. O objetivo deste trabalho é verificar, em uma revisão sistemática da literatura, o estado da arte da avaliação da mucosite oral em crianças sob tratamento oncológico. Estudos mostraram que a avaliação é fundamental para estabelecer as estratégias de tratamento, que protocolos e escalas utilizadas são inconsistentes e que inexiste um sistema viável e válido para pontuar a mucosite infantil causada por câncer, impedindo uma intervenção terapêutica mais efetiva. Concluiu-se que a Children's International Mucositis Evaluation Scale vem sendo a escala de maior aceitabilidade e validade por seu critérios.

Palavras-chave: Neoplasia; Quimioterapia; Mucosite; Protocolo.

\begin{abstract}
Cancer is a public health problem in the world and, in children, the incidence of increase is around $1 \%$ per year. Despite its effectiveness, antineoplastic therapy causes effects of significant toxicity, often causing the patient to be hospitalized, difficult to continue with oncotherapy or not readjusted to the treatment for the non-progression of the disease. Oral mucositis is one of the most common deleterious effects; it causes pain, affects speech and nutrition, and makes the
\end{abstract}


patient susceptible to sepsis. In children, the frequency of oral mucositis is around $65 \%$. Its manifestations are evaluated using different scales and treated with different protocols. The objective of this work is to verify, in a systematic literature review, the state of the art in the evaluation of oral mucositis in children undergoing cancer treatment. Studies have shown that assessment is essential to establish treatment strategies, that protocols and scales used are inconsistent, and that there is no viable and valid system to score childhood mucositis caused by cancer, preventing a more effective therapeutic intervention. It was concluded that the Children's International Mucositis Evaluation Scale has been the scale with the greatest acceptability and validity according to its criteria.

Keywords: Neoplasm; Chemotherapy; Mucositis; Protocol.

\section{Resumen}

El cáncer es un problema de salud pública en el mundo y, en los niños, la incidencia de aumento es de alrededor del $1 \%$ anual. A pesar de su eficacia, la terapia antineoplásica provoca efectos de importante toxicidad, provocando a menudo la hospitalización del paciente, dificultad para continuar con la oncoterapia o no readaptación al tratamiento por la no progresión de la enfermedad. La mucositis oral es uno de los efectos deletéreos más comunes; causa dolor, afecta el habla y la nutrición y hace que el paciente sea susceptible a la sepsis. En los niños, la frecuencia de la mucositis oral es de alrededor del 65\%. Sus manifestaciones se evalúan utilizando diferentes escalas y se tratan con diferentes protocolos. El objetivo de este trabajo es verificar, en una revisión sistemática de la literatura, el estado del arte en la evaluación de la mucositis oral en niños sometidos a tratamiento oncológico. Los estudios han demostrado que la evaluación es fundamental para establecer estrategias de tratamiento, que los protocolos y escalas utilizados son inconsistentes y que no existe un sistema viable y válido para puntuar la mucositis infantil por cáncer, evitando una intervención terapéutica más eficaz. Se concluyó que la Children's International Mucositis Evaluation Scale ha sido la escala con mayor aceptabilidad y validez según sus criterios.

Palabras clave: Neoplasia; Quimioterapia; Mucositis; Protocolo.

\section{Introdução}

O câncer é problema de saúde pública em todo o mundo. (Hashemi et al., 2015). Em crianças e adolescentes, tem incidência de aumento de $1 \%$ a cada ano. Um relatório recente estimou que, em 2017, 10.270 crianças de até 14 anos foram diagnosticados com câncer e que 1.190 delas morreram.(Garrocho-Rangel et al., 2018).

Para a World Health Organization (WHO), a administração das doenças malignas requer frequentemente a utilização de terapias com efeitos bastante tóxicos para o paciente. Para se verificar a ação específica, comparam-se os efeitos da diversidade de tratamentos com seu custo potencial quanto à toxicidade. Dessa forma, o conhecimento dos efeitos tóxicos das drogas usadas é aspecto essencial a ser examinado, paralelamente aos resultados esperados do tratamento. (WHO, 1979, p. 14 -16). Essas malignidades causam complicações que podem ser tanto consequência direta delas, como efeito adverso do tratamento adotado para combatê-las (radioterapia, quimioterapia ou os dois). (Hashemi et al., 2015).

Apesar de sua alta eficácia, a quimioterapia provoca muitos efeitos de significativa toxicidade sobre os pacientes, incluindo as crianças. (Devi et al., 2019) Isso ocorre porque as terapias antineoplásicas, ao tempo em que combatem as células tumorais, também lesionam células normais. (Paiva et al., 2010) Além da toxicidade das drogas, outros aspectos devem ser considerados, como a radioterapia associada, a saúde geral do paciente e sua susceptibilidade aos agentes do tratamento. (Castro et al., 2013)

Os efeitos colaterais da terapia antineoplásica devem ser tratados paralelamente à doença. (Paiva et al., 2018). São variados e, de modo geral, seu acometimento não se limita a um tipo, dependendo da terapia adotada no caso - quimioterapia, radioterapia ou os dois - e das condições particulares do paciente. Entre os principais efeitos encontram-se as complicações orais - mucosite, xerostomia, infecções fúngicas, virais e bacterianas, osteorradionecrose e outras - que podem se manifestar de forma aguda ou tardia. Elas interferem na integridade e na função da cavidade oral e, em pacientes pediátricos, podem comprometer até as áreas da formação dentária e da formação óssea. (Paiva et al., 2018). A mucosite oral é um evento biológico causado por fatores do tratamento e fatores relacionados ao paciente (Otmani et al., 2011), entre eles: o tipo de câncer, o grau de supressão da medula óssea (Bulut; Tüfekci, 2016), a toxicidade do medicamento, a dosagem, o intervalo entre os ciclos de tratamento, a associação da radioterapia, a saúde geral do paciente, suscetibilidade aos agentes quimioterápicos, condição dentária e higiene 
oral do paciente (Castro et al., 2013 ). Outras condições do paciente também são citadas, como: idade, índice de massa corporal, gênero, alterações na produção salivar e trauma da mucosa, foram relatados como fatores de influência de desenvoovimento da mucosite. Os fatores genéticos têm papel de risco na toxicidade, e os determinantes genéticos do risco de mucosite englobam os genes que regulam a disponibilidade de metabólitos ativos de drogas quimioterápicas. (Mendonça et al., 2015) A mucosite também pode ser agravada por aspectos locais. (Paiva et al., 2018)

Esses fatores estão entre os que determinam a suscetibilidade do paciente à mucosite oral, como demonstram $40 \%$ das terapias anticancegínenas de dose padrão e $50 \%$ das terapias de alta dose. Esse percentual aumenta em até $90 \%$ quando há quimioterapias combinadas para tratamento do câncer infantil. (Bulut; Tüfekci, 2016)

Muitas vezes, esses efeitos podem resultar em hospitalização do paciente, dificultar a continuidade da oncoterapia ou impedir a continuidade do tratamento adequado ante a necessidade de se reduzir as dosagens ou a modificação do esquema em relação ao protocolo para inibir a progressão da doença. De modo mais violento, os efeitos podem aumentar a morbidade e a mortalidade de pessoas em tratamento oncológico. (Hashemi et al., 2015; Devi et al., 2019) O adequado manejo dessas condições pode prevenir o surgimento de comorbidades e evitar a interrupção da quimioterapia. (Garrocho-Rangel et al., 2018).

No geral, a prevalência de mucosite oral causada pela quimioterapia varia de 30\% a 75\% (Aggarwal et al., 2014). Entre os pacientes pediátricos com câncer, a taxa é três vezes maior do que em adultos (Bulut; Tüfekci, 2016), sendo um dos principais efeitos da quimioterapia citotóxica (Figliolia et al., 2008). Crianças abaixo de 12 anos têm 90\% de chance de desenvolver a mucosite (Devi et al., 2013), podendo chegar a quase 100\% (Argelagós et al., 2014). Em crianças com câncer hematológico, o risco é duas ou três vezes maior de desenvolver problemas orais, quando comparado às crianças com tumores sólidos. Estudos com uma amostra de 26 crianças de idade de 1 a 14 anos, com neoplasias hematológicas e submetidas à quimioterapia, verificaram que $61 \%$ delas tiveram mucosite. Outra pesquisa na Indonésia, com 30 crianças, demonstrou que $67 \%$ delas tiveram e um terceiro estudo comprovou que 50\% das crianças com leucemia tiveram mucosite após a quimioterapia (Devi et al., 2019). Por isso, pacientes com câncer, antes de se submeterem à terapia oncológica, devem fazer um exame odontológico completo para verificar suas condições orais e tratar eventuais lesões dentárias e que podem causar mais complicações durante o tratamento. $\mathrm{O}$ atendimento e a atenção odontológica podem solucionar necessidades e estabelecer um esquema preventivo (Argelagós et al, 2014). Em qualquer hipótese, a prevenção e o tratamento da mucosite causada pelas terapias oncológicas se enquadra na perspectiva orodontológica (Singh et al., 2019).

Algumas estratégias são essenciais para verificar, de modo mais seguro, as dificuldades relativas às terapias de combate à mucosite oral. No caso de crianças com câncer, não há cooperação nos exames da cavidade oral. Além disso, o fato de alguns sintomas funcionais poderem se vincular a um comportamento infantil específico e não a uma etiologia associada à mucosite representa outro dificultador (Jacobs et al., 2013). Assim, se por um lado a vigilância e o monitoramento contínuos do estado oral de pacientes pediátricos com câncer são fundamentais para a prevenção e para o tratamento das afecções causadas pela mucosite oral nas mucosas e na língua, por outro, segundo a literatura da odontopediatria, não há consenso quanto às estratégias ou drogas agentes específicas capazes de solucionar o problema (Garrocho-Rangel et al., 2018). Até recentemente, não havia agentes aprovados capazes de reduzir a incidência nem a duração da mucosite oral (Stiff et al., 2006).

No momento, não há um tipo de tratamento padrão para mucosite oral (Kuhn et al., 2009). Embora se venha enfatizando a necessidade de sua prevenção sempre que possível, poucos trabalhos têm examinado a prevenção, a redução da incidênciada mucosite e sua gravidade em crianças que se submetem às terapias oncológicas (Allen et al., 2010). Mas não só é escassa a literatura nesse sentido, como também são mal compreendidos os exatos fatores de risco da doença nessa população infantil, mesmo com a grande probabilidade de desenvolvimento dessa complicação (Mendonça et al., 2015). Logo, a inexistência de um sistema viável e válido para avaliar e pontuar o grau da mucosite oral em crianças tornou-se um obstáculo à intervenção mais efetiva na mucosite oral proveniente de câncer infantil (Jacobs et al., 2013). 
Desse contexto, surge a seguinte questão: ante as lacunas apontadas anteriormente, que instrumentos vêm sendo utilizados de forma segura para avaliar o grau de mucosite oral em crianças, decorrente de tratamento antineoplásico? O objetivo deste trabalho é verificar o estado da arte dos instrumentos de avaliação da mucosite oral em crianças submetidas a tratamento oncológico, quanto à segurança na detecção do respectivo grau e à aceitabilidade.

\subsection{Mucosite oral}

A cavidade oral é o único ambiente no qual peptídeos antimicrobianos desempenham o papel essencial de manutenção da saúde e podem receber aplicações terapêuticas. Defencinas, histatina e outros peptídeos e proteínas antimicrobianas desempenham papéis distintos de forma sobreposta na manutenção da saúde oral e na prevenção da aderência bacteriana, fúngica e viral e infecção. Porém, alguns polimorfismos genéticos podem alterar a função de defensinas e causar susceptibilidades à mucosite oral. Polimorfismos dos genes podem ser causa da suscetibilidade genética às toxicidades induzidas pela quimioterapia. Pesquisas genômicas buscam identificar caminhos fundamentais de sinalização dos elementos envolvidos nos processos patológicos (Kuhn et al., 2009).

A mucosite oral é uma doença comum, considerada efeito colateral e debilitante da quimioterapia. Sua taxa de incidência é de $40 \%$ a 100\%, conforme o tipo de malignidade, o esquema quimioterápico, o respectivo medicamento, a idade do paciente, os cuidado bucais e a contagem de neutrófilos (Hashemi et al., 2015).

Houve um progresso significativo referente à compreensão da patobiologia da mucosite. Se anteriormente ela era entendida como simples reflexo do dano epitelial direto, causado pela terapia citotóxica, agora é vista como fenômeno de grande complexidade que afeta também o tecido conjuntivo (Mendonça et al. 2015). Pesquisas recentes indicam que a mucosite oral é resultado de um processo patológico (Otmani et al., 2011), havendo quem considere que a microflora oral tem papel secundário nessa patogênese (Mendonça et al. 2015).

A fisiopatologia da mucusite oral é dinâmica e multifatorial, e seu desenvolvimento se dá em cinco fases: iniciação, regulação positiva e geração de mensagem, ampliação de sinais, ulceração e cura. A iniciação é seguida de danos ou não ao DNA (Castro et al., 2013). Na resposta à fase de geração de mensagem, a ativação de fatores de transcrição, como o fator nuclear kappa B, causa um aumento tanto de citocinas pró-inflamatórias local como interleucina IL-6 e fator de necrose tumoral. Os eventos biológicos que ocorrem promovem reações em cascata que ampliam o dano ao tecido (Mendonça et al., 2015) e desencadeiam um processo destrutivo, com o epitélio oral podendo se decompor em úlcera (fase de ulceração) (Castro et al., 2013). As bactérias orais colonizam os tecidos conjuntivos desnudados, e os componentes da parede celular ativam os macrófagos para produzir citocinas inflamatórias adicionais (Mendonça et al., 2015). O processo de cura é dinâmico biologicamente, com a matriz extracelular da submucosa estimulando a migração e a proliferação de células epiteliais (Castro et al., 2013). As bactérias que colonizam ulcerações podem aumentar a gravidade do caso e retardar a cicatrização (Mendonça et al., 2015). Em pacientes neutropênicos, as bactérias podem invadir o sistema circulatório e desencadear bacteremia e sepse. Após a interrupção desse processo, dá-se a cura e o epitélio parece normal novamente, embora possa haver alterações contínuas, predispondo o paciente a complicações futuras (Mendonça et al., 2015).

A mucosite oral inclui todas as lesões (eritema, inflamação, úlcera, necrose) sintomáticas das membranas mucosas no sentido amplo e da mucosa orofaríngea em particular (Bourdelin et al., 2015). Essas lesões se apresentam, de modo geral, como áreas atróficas ou eritematosas, às vezes incluindo manchas brancas descamativas na mucosa oral que depois evoluem para úlceras dolorosas (Figliolia et al., 2008).

Na mucosite causada pela quimioterapia, os sintomas são vistos inicialmente entre o $3^{\circ}$ e o $5^{\circ}$ dias após o início do tratamento e alcançam o auge cerca de 7 a 14 dias depois, com seu curso durando normalmente em torno de três semanas (Hashemi et al., 2015). A cicatrização começa a partir do $14^{\circ}$ dia se não houver infecção. Caso se desenvolva uma infecção, os 
primeiros sinais - vermelhidão, edemas e lesões - são vistos na mucosa e se transformam em úlceras na boca (Bulut; Tüfekci, 2016).

A contaminação bacteriana das úlceras da mucosa é um forte fator de risco de grave septicemia. Estudos realizados entre 2007 e 2010 identificaram um foco oral entre $25 \%$ e 50\% dos casos de septicemia grave, o que pode, principalmente, aumentar o índice de mortalidade. A septicemia também prolonga a estada de crianças em hospital, amplia o tempo de tratamento oncológico e aumenta os respectivos custos. Por todas essas razões, a mucosite é a maior complicação da quimioterapia e pode não só ser curada como prevenida tão rápida quanto ocorra ou possa ocorrer (Bulut; Tüfekci, 2016). A mucosite superinfectada varia de 6 a 30\% (Bourdelin et al., 2015).

A mucosite oral provoca diminuição na produção de saliva, causa sangramento, dor e possibilita o surgimento de bactérias, de vírus e de infecções fúngicas (Bulut; Tüfekci, 2016), além de disfagia, de dificuldades de beber, de comer e de engolir e outras (Garrocho-Rangel et al., 2018). Em crianças, tais efeitos fazem com que elas não consigam nem mesmo ingerir alimentos líquidos, sendo mais das vezes necessária a nutrição parenteral total (Bulut; Tüfekci, 2016). A dor e o desconforto são os efeitos mais comuns da mucosite e, em casos mais graves, podem resultar em desidratação, em desnutrição e em latência vitalícia nos gânglios neurais sensoriais com reativações periódicas. Vários eventos podem precipitar sua reativação, incluindo infecções intercorrentes (Aggarwal et al., 2014). As dores podem afetar a fala negativamente, as funções orais e a qualidade de vida dos pacientes, resultando, não raras vezes, na hospitalização do paciente e até no impedimento da continuidade do tratamento oncológico adequado, já que a dosagem da quimioterapia deve ser reduzida ou modificada em relação ao protocolo para inibir a progressão da doença. De modo ainda mais violento, ela pode aumentar a morbidade e a mortalidade (Hashemi et al., 2015), além de aumentar os níveis de estresse do paciente, principalmente o pediátrico. Por todas essas complicações, a detecção e principalmente a avaliação dessas complicações do tratamento do câncer pediátrico são essenciais (Paiva et al., 2018).

\subsection{Intervenções terapêuticas mais utilizadas}

Mucosite é uma doença apoiada em opções de tratamento e de cuidados de suporte. As medidas preventivas permanecem limitadas até hoje (Bourdelin et al., 2015), e o tratamento principal é de suporte, consistindo na utilização de uma vasta lista de medicamentos que abrangem da higiene oral à analgesia (Kuhn et al., 2009).

Pesquisas sobre os tipos de intervenção na mucosite oral causada pelo câncer apontam o uso de vários tipos de medicação, de terapias e de protocolos, intensivos ou não, revelando-se um campo vasto de investigação que passa por fatores que vão além dos objetivos deste trabalho, a exemplo da mucosite como ciclo prévio de tratamento da gastrite e da mucosite associada a outras importantes condições de saúde e custos econômicos (Kuhn et al., 2009).

Vários protocolos de cuidados orais intensivos, de uso de agentes antimicrobianos, de anti-inflamatórios e agentes citoprotetores, de fatores de crescimento, de agentes naturais e homeopáticos e de anestésicos locais são implementados (Castro et al., 2013). Vitaminas, citocinas, reguladores do sistema imunológico e medicamentos fitoterápicos, entre outros (Hashemi et al., 2015), assim como amifostina, gluconato de clorexidina, mastigação de gelo, antibióticos, anestésicos tópicos e protocolos diversos de higiene bucal também são muito utilizados (Garrocho-Rangel et al., 2018). Na literatura de enfermagem, são limitados os estudos sobre o tratamento da mucosite em crianças com câncer, e não há um agente padrão-ouro para o respectivo controle. O padrão mais comum e ainda atual é o da higiene oral e se refere a gargarejos com uma solução de água morna, sal e bicarbonato de sódio quatro vezes ao dia, além dos cuidados bucais básicos. Outro padrão sugerido é a higiene bucal como prevenção, avaliação oral diária, higiene bucal a cada quatro horas por dia, aplicação de uma solução de clorexidina $0,12 \%$ com bastão de esponja descartável, escova macia de dente e aplicação de gel hidratante na membrana mucosa e lábios e limpeza de pasta de dente residual e secreções com seringa de irrigação ou esponja (Devi et al., 2019). 
Estudos experimentais avaliaram a eficácia de diferentes protocolos de higiene bucal na redução de complicações orais causadas pela quimioterapia e apontaram o uso da clorexidina e de enxaguatórios bucais suaves como eficazes e mais acessíveis economicamente. Porém, a Multinational Association for Supportive Care in Cancer não recomenda o uso de enxágue bucal com clorexidina para a prevenção da mucosite em pacientes com tumores sólidos de cabeça e pescoço e em tratamento radioterápico (Allen et al., 2010). A palifermina, que é uma forma truncada e recombinante de crescimento de queratinócitos humano, diminui a gravidade e a duração da mucosite oral após terapia intensiva contra câncer hematológico em adultos ( Funato et al., 2018). Ela foi testada em animais e auxilia a proteção dos tecidos da mucosa contra os efeitos da irradiação. Em 2004, o Food and Drug Administration (FDA) dos Estados Unidos aprovou o uso da palifermina para reduzir a incidência e a duração da mucosite oral grave em pacientes adultos sob terapia mielotóxica (Stiff et al., 2006). Mas em crianças, cujas dosagens de quimioterapia são altas e com grave mucosite oral, a palifermina é pouco indicada como agente profilático, haja vista a falta de dados sobre sua eficácia e segurança (Funato et al., 2018; Stiff et al., 2006).

Outra estratégia de combate à mucosite é a crioterapia, com resfriamento rápido da cavidade oral. A estratégia provoca vasoconstrição local, reduzindo o fluxo sanguíneo no local e fazendo com que o agente tóxico em circulação alcance menos a mucosa oral. Se os agentes têm vida curta e são administrados via injeção, a crioterapia pode minimizar a potencialidade dos efeitos citotóxicos locais da droga na cavidade oral. A crioterapia não tem eficácia se o tratamento implementado for por infusão contínua e também causa desconforto ao paciente com o gelo na boca. Uma revisão sistemática da literatura de 2007 concluiu que a amifostina, enzimas hidrolíticas e chips de gelo promoveram uma significativa diferença na prevenção ou na redução da gravidade de mucosite oral, quando comparados a medicamentos placebo e ao não tratamento (Alen et al., 2010).

Em 2009, surgiu uma alternativa para a prevenção da mucosite oral provocada pela terapia anticancerígena de alta dose: a solução eletrolítica Caphosol com íon supersaturado, cálcio e fosfato. Estudos avaliaram o uso dessa solução e identificaram fatores de risco que preocupam pacientes que usam o metotrexato. Dessa forma, embora a utilização dessa solução no manejo da prevenção da mucosite não tenha sido definida, já parece limitada quanto à respectiva redução. Trata-se de uma opção pouco avaliada na prática clínica (Bourdelin et al., 2015).

Alguns estudos indicam a capacidade do uso tópico do mel de reduzir a incidência de mucosite oral decorrente da radioterapia e da quimioterapia na cabeça e no pescoço de pacientes em geral, submetidos à terapia antineoplásica. O peróxido de hidrogênio do mel e sua grande capacidade antioxidante são responsáveis por sua ação antimicrobiana, antifúngica, antiviral, antiinflamatória, cicatrizante e protetora contra infecções (Singh et al. 2019).

A terapia com laser de baixo nível vem demonstrando ser uma opção para tratamento da mucosite, tanto na prevenção como no tratamento em pacientes pediátricos submetidos a tratamento oncológico, inclusive com uso de metotrexato (Castro et 1., 2013). O laser é um método de irradiação simples não traumático. Estudos sugerem que ele promove a estimulação metabólica na cicatrização de feridas, o aumento do tecido de granulação e da epitelização precoce, o aumento da proliferação de fibroblastos e a neovascularização aprimorada. Porém, sua eficácia não foi ainda totalmente comprovada (Kuhn et al., 2009). Os resultados são positivos pela facilidade de uso, pela ausência de efeitos colaterais e pelo baixo custo do equipamento (Castro et al., 2013).

Em resumo, há duas situações: centros especializados para qualquer tipo de câncer usam protocolos diferentes de tratamento (Figliolia et al., 2008), e ensaios clínicos sobre essas intervenções demonstraram resultados inconsistentes, ou seja, sua eficácia não foi comprovada com precisão (Castro et al., 2013).

Muitas pesquisas e estudos vêm sendo realizados no mundo inteiro para desenvolver um método eficaz de manejo da mucosite causada pela quimioterapia em crianças. Porém, ainda não se obteve um regime de tratamento específico para esse manejo (Singh et al., 2019). A avaliação do resultado dos protocolos utilizados foi inconsistente, sem nenhum padrão de eficácia comprovado (Castro et al., 2013). Não há um método terapêutico padronizado único para prevenir e tratar a mucosite; o tratamento varia conforme as instituições (Singh et al., 2019), como referido. 
A mucosite oral decorrente de quimioterapia deve ser sistematicamente avaliada e analisada de modo prospectivo em centros especializados para o tratamento de câncer, para estabelecer o grau de toxicidade dos medicamentos quimioterápicos e para melhorar a qualidade de vida dos pacientes com base em mais abordagens terapêuticas e profiláticas, eficazes para prevenção da sua ocorrência (Figliolia et al., 2008). Diferenças na gravidade da mucosite entre pacientes tratados com o mesmo regime de quimioterapia foram relacionados à predisposição genética para mucotoxicidade. Apesar dessas descobertas, o espectro da patogênese e os fatores de risco exatos da mucosite oral causada pela terapia antiancerígena ainda são mal compreendidos, especialmente em crianças com câncer (Otmani et al., 2011). A avaliação da mucosite oral é importante para comparar estratégias de tratamento e de prevenção. Ensaios clínicos com foco na prevenção e no tratamento da mucosite concluíram que uma e outro requerem instrumentos confiáveis, válidos e fáceis de utilizar (Tholinson et al., 2009).

\subsection{Escalas de avaliação da mucosite}

Anteriormente, o nível da mucosite era avaliado por meio de escalas elaboradas pela Organização Mundial da Saúde (OMS), dos critérios do Instituto Nacional do Câncer, em sua terminologia relativa aos evenos adversos, e da escala de Avaliação da Mucosite Oral. Esses instrumentos avaliam a mucosite com base na observação dos profissionais de saúde, à vista do exame clínico, sem medir pontualmente a mucosite em pacientes oncológicos pediátricos (Paiva et al., 2018).

Argelagós et al. (2014), por meio de uma revisão sistemática de pesquisas do período de 1998 a 2014, analisaram seis protocolos de atenção odontológica preventiva na oncologia pediátrica, quanto à saúde oral e à mucosite. Observaram que não há unanimidade entre os critérios, apesar de a clorexidina, sem álcool, aparecer como o produto mais usado, sob forma de enxague ou não, seguida da escovação dental e do uso do bicarbonato de sódio e outros de menor expressão.

A World Health Organization (WHO), Organização Mundial da Saúde (OMS), propôs uma classificação dos efeitos da toxicidade da terapia oncológica, classificando-os em subagudos, crônicos ou posteriores, diferentes da classificação associada às modalidades de tratamento. Os efeitos tóxicos subagudos possibilitam comparar a toxicidade entre os programas de tratamento, permitem quantificar e analisar os dados da toxicidade e alinhar o tratamento ao programa da terapia oncológica. Para tanto, a significância clínica - suave, moderada, severa e risco de vida - deve ser avaliada. Os efeitos crônicos ou posteriores são mais comuns e tendem a se estender por um bom tempo. A severidade de suas manifestações é mais difícil de quantificar do que a dos efeitos subagudos. A WHO sugere os seguintes critérios para avaliar os efeitos crônicos: área ou órgão afetado, tempo de manifestação para identificar as causas, natureza da toxicidade ou da incapacidade produzida, magnitude dos sintomas, impacto dos efeitos no desempenho do paciente, tipo de terapia oncológica utilizada e a respectiva resposta do paciente. Os pacientes devem ser avaliados anualmente (WHO, 1979).

No ambiente clínico, o uso de sistemas de avaliação sem pontuação e não validados em crianças pequenas pode fornecer dados incorretos e levar, consequentemente, a tratamentos inadequados e muitas vezes caros. Várias escalas de avaliação da mucosite oral infantil são inadequadas porque, como dito, crianças muito pequenas não têm condição de cooperar no processo longo de exames. É o caso da Escala de Avaliação de Mucosite Oral da OMS e de outros instrumentos muito usados na prática clínica, como o Guia de Avaliação Oral. No geral, esses instrumentos podem contribuir para avaliar a saúde e a higiene bucal, mas muitos de seus itens não abrangem especificamente a mucosite. A escala da WHO se baseia na impossibilidade ou dificuldade do paciente para comer e para beber como critério de avaliação do maior grau de mucosite. Mas crianças com câncer podem não conseguir comer ou beber por motivos não associados à mucosite. Já a escala do National Cancer Institute - Common Toxicity depende da capacidade de hidratação oral das crianças para classificar os sintomas funcionais da mucosite (Tolinson et al., 2009).

A Escala de Avaliação de Mucosite Oral da OMS, a mais usada, caracteriza a mucosite oral em graus que vão de zero a 4, segundo a aparência e critérios: grau zero, mucosas sem alteração; grau 1, presença de dor ou de eritema; grau 2, eritema e 
úlceras; grau 3, úlceras, com tolerância só à dieta líquida; grau 4, úlceras, sem tolerância a qualquer tipo de alimentação. Os graus 1 e 2 caracterizam a mucosite oral de leve a moderada, e os graus 3 e 4, mucosite oral grave (Schubert et al.1992).

A escala do National Cancer Institute - Common Toxicity Criteria avalia a toxicidade oral por meio de sinais objetivos, como eritema e ulceração, associados a critérios funcionais e subjetivos, como dor, alteração da capacidade de deglutir. Com base nisso, foram estabelecidos: grau zero, ausência de mucosite; grau 1, presença de úlceras sem dor; grau 2, presença de eritemas dolorosos; grau 4, ulcerações graves, incluindo necessidade de intubação profilática ou de nutrição parenteral; grau 5, toxicidade relacionada ao óbito (Sonis et al., 1999).

O Oral Mucositis Index avalia a mucosite oral com base nos graus: 1) pouca ou nenhuma variação das membranas, como ulceração e liquenoides na língua, condição do palato, da gengiva, edema na língua e outros; 2) variação baixa, como atrofia no palato e na gengiva, pseudomembranas na mucosa e nos lábios e edema nos lábios e nas mucosas labiais; 3) base em componentes principais, como atrofia, eritema e edema das mucosas, da língua e do assoalho da boca (Schubert et al.1992).

Além dessas, ainda podem ser citadas: o Oral Mucositis Daily Questionnaire (OMDQ), que avalia a mucosite oral e seu processo com base nos relatos do paciente sem a vista do profissional. O paciente relata sua percepção sobre sua saúde de uma maneira geral, presença de diarreia e intensidade da dor na cavidade oral e orofaringe, e o impacto desta na realização de atividades como engolir, beber, alimentar-se, falar e dormir. Nas primeiras versões, os resultados demonstraram que o uso do OMDQ na avaliação da mucosite oral foi positivo quando comparado com as avaliações clínicas no tratamento com a palifermina (indicada para adultos). Em outros testes, o questionário foi sendo reduzido. Para a maior parte das questões avaliativas, a pontuação mair aponta piora na gravidade dos sintomas da mucosite ou maior interferência da mucosite nas atividades funcionais orais. O instrumento não foi adaptado para o Brasil (Stiff et al., 2006).

Em resumo, várias escalas foram criadas visando mensurar a mucosite oral em crianças, sem que se tenha verificado se alguma delas atende melhor a um propósito específico da mucosite e detém requisitos de validade, de confiabilidade, de viabilidade e de preferência (Tholinson et al., 2010b).

Em 2009, no Canadá, foi desenvolvida uma escala para a mensuração da mucosite oral em crianças submetidas a terapias oncológicas, para avaliar o respectivo impacto sobre as funções orais, desde a hidratação oral, a deglutição e a alimentação, até a fala. Trata-se da Child's Internacional Mucositis Evaluation Scale (Tholinson et al., 2010a).

\subsection{Child's Internacional Mucositis Evaluation Scale (ChiMES)}

Do ponto de vista biológico, intervenções eficazes com pessoas adultas nem sempre têm o mesmo efeito em crianças. As diferenças podem ser a alteração da farmacocinética e da farmacodinâmica pela idade do paciente e por sua falta de cooperação, principalmente do paciente em alguns tipos de terapia interventiva, além de o efeito da toxicidade das terapias também poder ser diferente em adulto e em criança (Sung et al., 2014).

As escalas utilizadas para avaliar a mucosite em crianças submetidas à terapia oncológica são consideradas inadequadas para uso específico nesse público-alvo, porque além de elas não cooperarem para a realização de exames longos, como a Escala de Avaliação de Mucosite Oral da OMS, esses instrumentos foram desenvolvidos para uso em adultos. Da escala da OMS, consta o item "incapacidade para ou beber" como critério de atribuição do maior grau de mucosite, sendo que essa incapacidade pode se dar por razões não relacionadas à mucosite, mas sim à própria terapia oncológica. Outras escalas, como o Guia de Avaliação Oral, podem avaliar bem a saúde e higiene bucal, mas muitos de seus itens não são específicos da mucosite, como lábios secos e voz. Todos esses pontos, que limitam a avaliação da mucosite oral em crianças com câncer, foram observados por um grupo de especialistas que concluiu não ser nenhuma dessas escalas adequada para determinar o tratamento mais eficaz da mucosite pediátrica. A realização de ensaios clínicos com foco na prevenção e no tratamento mucosite exige instrumentos confiáveis, válidos, sensíveis e fáceis de utilização. O uso de um sistema de pontuação não validado em crianças pequenas na 
prática clínica pode fornecer informações incorretas e, consequentemente, levar à implementação de um tratamento inadequado e dispendioso (Tomlinson et al., 2009a).

Essa lacuna na literatura demonstrou a necessidade de se desenvolver uma nova forma de avaliar a mucosite pediátrica, com itens gerados especialmente para a avaliação em crianças e com conteúdo de fácil compreensão (Jacobs et al., 2013). De início, revisou-se a Escala de Avaliação de Mucosite Oral da OMS, para verificar os itens baseados na literatura que deveriam ser considerados na avaliação da mucosite oral daquele público-alvo. Depois, instituiu-se um grupo focal de pesquisa, constituído de especialistas em mucosite pediátrica de instituições internacionis e por uma equipe multidisciplinar, com a finalidade de obter opiniões consensuais quanto aos itens do instrumento. Na sequência, concluiu-se que a nova escala deveria incorporar três componentes essenciais a qualquer instrumento de avaliação da mucosite oral: a dor, quanto ao grau e à medicação usada para combatê-la; a função ou capacidade de engolir, de comer e de beber, quanto ao nível de desempenho ou de dificuldade; a aparência da mucosa oral, com deteç̧ão visual de ulcerações (Tomlinson et al., 2009a). Especificamente quanto à dor, três abordagens distintas têm sido utilizadas: autorrelato, quanto ao grau percebido; avaliação e observação do comportamento da face na ocorrência da dor, respostas motoras e o choro; respostas fisiológicas à percepção dolorosa, como aumento da frequência cardíaca e queda da saturação de oxigênio (Tomlinson et al.,2010b). Obteve-se a uma versão inicial do instrumento que se chamou de Child's Internacional Mucositis Evaluation Scale (ChiMES) (Tomlinson et al., 2009a).

Na sequência, identificou-se a necessidade de nova análise pediátrica da avaliação da mucosite. Foram então gerados itens com base na literatura e resultados consensuais foram obtidos com a técnica de grupo nominal (Tomlinson et al., 2010a). Critérios de quatro escalas entre as mais usadas foram sistematicamente revisados e resumidos, buscando avaliar, por meio do autorrelato, a intensidade da dor nas crianças e verificar a confiabilidade e a validade dos critérios, comparando a utilidade e a preferência desses critérios entre as escalas (Tomlinson et al. 2010b). Esse processo de criação englobou as etapas: 1 - revisão das escalas de avaliação da mucosite oral em uso, inclusive a utilizada para a população adulta; 2 - identificação dos aspectos mais desafiadores e das potenciais soluções para avaliação da mucosite oral em crianças; 3 - estabelecimento de itens baseados na literatura referente à mucosite oral, passíveis de utilização em instrumentos de avaliação em crianças; 4 - geração de itens para avaliar a mucosite oral sob forma de instrumento, com a técnica de grupo nominal; 5 - criação do instrumento de avaliação da mucosite oral com o conteúdo gerado; 6 - teste de compreensibilidade, aceitabilidade e conteúdo validade e refinamento do instrumento da criança e dos pais relatórios (Tomlinson et al., 2010a).

O passo seguinte se refereriu ao desenvolvimento da etapa 6, que foi desenvolvida por meio de métodos de compreensibilidade de conteúdo. Tratava-se da aceitabilidade desse aspecto, cujos testes avaliaram o processo desde o esboço inicial da ChIMES. Para testar a compreensibilidade, pediu-se que os participantes da pesquisa (pais, filhos crianças e jovens) avaliassem cada questão por meio da pontuação de cinco graus que iam de "compreensão muito difícil" a "compreensão muito fácil”, solicitando sugestões de melhoras. A revisão de validade de conteúdo passou a acomodar itens voltados para a compreensão das crianças, para que elas entendessem o que lhes estava sendo pedido. Pais também se manifestaram por meio de pareceres positivos e de formulários de consentimento. A aceitabilidade geral foi avaliada pedindo aos participantes que avaliassem todo o questionário, com pontuação que verificava desde "muito difícil de completar" a "muito fácil de completar" (Tomlinson et al., 2010a).

A viabilidade e a validade da ChiMES foram testadas com base nos seguintes requisitos, respectivamente: teste-reteste, interexaminador e consistência interna; validade convergente e capacidade de resposta. Considerou-se importante a presença de acompanhante e dos pais para melhor identificar as respostas, conforme a idade da criança. Foram sete medidas de avaliação: 1intensidade de dor na boca ou na garganta; 2- consequências da dor na boca, na garganta e na capacidade de engolir; 3- efeitos da dor na boca, na garganta e na capacidade de comer; 4- interferência da dor na boca, na garganta e na capacidade de beber; 5utilização de medicação para a dor; 6- utilização de fármaco para dor na boca ou na garganta; 7-presença de ulcerações. Porém, 
como a dificuldade ou impossibilidade de alimentação em crianças pequenas podem seu causadas por outros problemas, como náusea e anorexia, a ChiMES possibilita que o respondente informe "não sei dizer"(Jacobs et al., 2013).

Os escores da ChiMES podem ser calculados de duas formas: total e percentual. No escore total, os pontos vão de 0 a 23, com medidas de 1 a 4, variando os graus entre 0 e 5, indicativos de ausência e pior grau, respectivamente, e as medidas de 5 a 7, a resposta "sim" vale um ponto e "não", recebe zero. Nas respostas "não sei dizer" a pontuação é zero. No escore percentual, o cálculo é feito com a divisão do escore total obtido pelo escore total máximo (que é 23), menos as respostas "não sei dizer" (subtraídas do escore máximo), multiplicado por 100. A mais alta pontuação corresponte ao pior grau da mucosite. No total, foram necessárias três iterações até se chegar à versão final da ChiMES. Em síntese, o novo instrumento de avaliação da mucosite oral em crianças gerou itens para a escala e avaliou a psicometria inicial sem perder de vista a compreensão do conteúdo, a validade e a aceitabilidade do instrumento pelos pais e pelas crianças com câncer (Jacobs et al, 2013). O estudo foi aprovado pelo Conselho de Ética em Pesquisa de The Hospital for Sick Children de Toronto, no Canadá e contou com o termo de consentimento informado dos familiares das crianças submetidas à testagem com a ChiMES (Tomlinson et al., 2010a).

Para serem usada em países diferentes do país de origem, as escalas necessitam não só de tradução, mas também de adaptação cultural. A ChiMES passou por esse processo na tradução para a língua portuguesa. Dois fluentes tradutores da língua inglesa traduziram o material do original, fazendo-se uma síntese das duas. Essa versão foi retrotraduzida para o inglês por dois falantes nativos de inglês, fluentes em língua portuguesa, e um dos autores da escala avaliou a retrotradução, visando à confirmação da manutenção dos significados dos itens e das respostas. Especialistas em semântica analisaram a equivalência conceitual e cultural da versão pré-final da escala, atribuindo pontos de representatividade. O índice de validação do conteúdo foi calculado por meio da soma do número com pontuação 3 e 4 dividido pelo número total de itens, sendo 0,80 o valor mínimo aceitável. O resultado final desse processo foram a versão autorrelatad em português brasileiro, a Escala Internacional de Avaliação de Mucosite em Crianças (ChIMES-BR) (Paiva et al., 2018).

\section{Materiais e Métodos}

Trata-se de uma revisão sistemática de literatura sobre a mucosite oral e as respectivas formas de tratamento em crianças submetidas a terapias oncológicas conforme orietanções Botelho et al. (2011). As pesquisas foram realizadas nas bases de dados PubMed e Scopus, primeiramente por meio dos seguintes descritores: crianças em quimioterapia, efeitos colaterais e prevenção. Após os resultados, os descritores foram refinados para mucosite, protocolos e terapias. Em uma terceira etapa, ante o surgimento da problemática de avaliação da mucosite oral nesse público-alvo, ou seja, ausência de protocolos específicos, fez-se uma revisão narrativa com o descritor avaliação da mucosite em crianças, para delimitar o tema. Os vários autores consultados e referidos foram fundamentais para o levantamento do estado da arte sobre o tema proposto. Já a parte que culmina com o aspecto atual desse estado da arte centrou-se em dois grupos de autores, Tolinson et al., com vários artigos, e Jacobs et al., que se detiveram na construção e na validação do novo instrumento de avaliação da mucosite em crianças em tratamento antineoplásico. Outros trabalhos encontrados sobre esse novo instrumento, além de relativamente escassos, tinham esses grupos de autores como referência. A metodologia utilizada tem como principal vantagem, em relação à revisão puramente narrativa, o maior rigor metodológico, além de ser mais específica e delimitada em relação ao tema abordado.

\section{Resultados e Discussão}

Inicialmente, os resultados foram unânimes quanto ao reconhecimento da mucosite oral em pacientes submetidos a tratamento anticancerígeno como evento desencadeado por fatores diversos, demonstrando que a abordagem desse tema não só é ampla, por aspectos distintos quanto à etiologia, causas, à avaliação e ao respectivo tratamento, como complexa, por envolver questões relativas ao paciente, à medicação, à doença e ao tratamento. 
Nos resultados, a etiologia da mucosite foi explicada do ponto de vista biológico e fisiológico, com evolução significativa quanto à respectiva compreensão. Se biologicamente ela se origina de polimorfismos genéticos que alteram a função de defensinas, promovem a susceptibilidade a efeitos tóxicos com processos patológicos e danificam o tecico conjuntivo (Kuhn et al., 2009; Otmani et al., 2011), fisiopatologicamente, seu desenvolvimento se dá em fases que vão desde a iniciação até a cura (Castro et al., 2013), com paralelos processos bacterianos que podem retardar essa cusa (Mendonça et al., 2015).

A pesquisa demonstrou que o contexto da complexidade dessa patologia é justificado pela diversidade de seus fatores desencadeantes. Sobre esses, enquanto Mendonça et al. (2015) citam as condições do paciente - idade, índice de massa corporal, gênero, alterações na produção salivar e trauma da mucosa, além das condições gerais no momento do tratamento -, Castro et al. (2013) se referem à toxicidade do medicamento, à dosagem, ao intervalo entre os ciclos de tratamento e a associação a outras terapias, e Bulut e Tüfekci (2016) mencionam o tipo de câncer e o grau de supressão da medula óssea. Adicionalmente, Paiva et al. (2018) declaram que a mucosite pode ser agravada por aspectos locais.

Epidemiologicamente, as informações indicaram que a mucosite oral em pacientes em tratamento antineoplásico tem percentuais também variados. Se quanto ao tipo de malignidade, ao esquema da quimioterapia, ao medicamento, à idade do paciente, aos cuidados bucais e à contagem de neutrófilos o percentual vai de 40\% a 100\% (Hashemi et al., 2015), especialmente quanto à idade, há discordância: 90\% de crianças abaixo de 12 anos podem desenvolver a mucosite (Devi et al., 2013), podendo chegar a quase $100 \%$ (Argelagós et al., 2014). Se na relação entre a forma da terapia antineoplásica (isolada ou combinada) e a susceptibilidade do paciente infantil à mucosite os percentuais não apontam diferenças muito significativas - 40\% das terapias com dose padrão, 50\% das terapias de alta dose e até 90\% em terapias combinadas (Bulut; Tüfekci, 2016) -, na prevalência geral, a ocorrência da mucosite tem variação ampla, 30\% a 75\% (Aggarwal et al., 2014), sendo três vezes mais em crianças (Bulut; Tüfekci, 2016).

$\mathrm{Na}$ investigação, foi mostrado que os efeitos da mucosite oral sobre o estado geral do paciente pediátrico também variam, com cada autor se referindo a um ponto de vista: comprometimento da formação dentária e da formação óssea (Paiva et al., 2018); aumento da morbidade e da mortalidade (Hashemi et al., 2015; Devi et al., 2019); hospitalização do paciente (Garrocho-Rangel et al., 2018); diminuição na produção de saliva, sangramento, dor e possibilidade de surgimento de bactérias, de vírus e de infecções fúngicas (Bulut; Tüfekci, 2016); surgimento de disfagia, dificuldades de beber, de comer e de engolir entre outras (Garrocho-Rangel et al., 2018). Os efeitos mais comuns citados foram os problemas de ingestão de alimentos e de líquidos (Bulut; Tüfekci, 2016), dor e desconforto (Aggarwal et al., 2014), e o destaque foi a contaminação das úlceras da mucosa por bactérias, como grande risco de septicemia grave (Bulut; Tüfekci, 2016) e de mucosite superinfectada (Bourdelin et al., 2015). Não houve qualquer discrepância entre os autores quanto a qualquer desses efeitos provocados pela mucosite oral em crianças submetidas a tratamento antineoplásico.

Relativamente à avaliação segura do grau da mucosite oral para a implementação de terapias efetivas de combate, a maioria dos autores consultados se referiu às escalas como os instrumentos mais utilizados, cada uma com limitações: a Escala de Avaliação de Mucosite Oral da OMS, a mais usada, avalia a mucosite oral em graus, segundo aparência, a alteração das mucosas e os efeitos sobre a deglutição (Schubert et al.1992); a escala do National Cancer Institute - Common Toxicity Criteria classifica a mucosite em graus segundo sinais objetivos (eritema e ulceração), associados ao comprometimento das funções e a aspectos e subjetivos (dor e capacidade de deglutir) (Sonis et al., 1999); o Oral Mucositis Index divide a mucosite oral em graus, conforme a aparência das membranas e das ulcerações e as condições gerais (palato, gengiva, língua e outros) (Schubert et al.1992); o Oral Mucositis Daily Questionnaire (OMDQ) classifica a mucosite oral e o respectivo processo de acordo com a percepção e o relato do paciente (engolir, beber, alimentar-se, falar e dormir sem que nenhum profissional averigue as mucosas) (Stiff et al., 2006). 
Sobre essas escalas para pacientes pediátricos, foi visto que esses instrumentos são inadequados por não avaliarem bem o grau do problema, já que não incluem itens específicos de mucosite e às vezes dependem de requisitos do paciente para sua utilização (Tolinson et al., 2009, 2009b).

Em relação às terapias usadas no combate à mucosite oral desencadeada por tratamento antineoplásico, os resultados apontaram um quadro ainda mais vasto de medicações e protocolos. De início, considerou-se que intervenções terapêuticas utilizadas em adultos não asseguram o mesmo resultado em crianças, devido às alterações farmacodinâmicas e farmacotécnicas da idade (Sung et al., 2014) e que, se por um lado, não existe uma terapia padronizada (Kuhn et al., 2009; Devi et al., 2019; Singh et al., 2019), por outro, pesquisas experimentais mostraram a eficiência de protocolos de higiene oral na redução do problema, indicando enxaguantes suaves e clorexidina pela eficácia e pelo acesso financeiro (Allen et al., 2010). Outros meios de combate à mucosite foram indicados pelos autores: reguladores imunológicos, medicação fitoterápica, citocinas e vitaminas (Hashemi et al., 2015); mastigação de gelo, gluconato de clorexidina, anestésicos locais, antibióticos e distintos protocolos de higiene oral (Garrocho-Rangel et al., 2018; Castro et al., 2013); agentes anti-inflamatórios, antimicrobianos e citoprotetores, homeopáticos e naturais (Castro et al., 2013); crioterapia (Alen et al., 2010); uso tópico do mel (Singh et al. 2019); solução eletrolítica Caphosol, com fosfato, cálcio e íon supersaturado (Bourdelin et al., 2015); palifermina (Funato et al., 2018) e terapia com laser de grau baixo (Castro et al., 2013).

Os resultados do estudo apontaram que esses esquemas terapêuticos têm limitações (Allen et al., 2010; Funato et al., 2018; Stiff et al., 2006), que sua eficácia não foi completamente comprovada (Kuhn et al., 2009; Castro et al., 2013), que a avaliação dos protocolos utilizados demonstrou inconsistência (Castro et al., 2013), que os fatores que causam a mucosite ainda não são muito bem compreendidos (Otmani et al., 2011), que em pacientes pediátricos não há cooperação deles nos exames orais (Jacobs et al., 2013) e que avaliações inadequadas dessa patologia levam a terapias nem sempre eficazes (Tolinson et al., 2009, 2009b).

Esse conjunto de conclusões, a inexistência de um padrão de terapia e as limitações das escalas utilizadas para se avaliar a mucosite levaram ao entendimento de que o obstáculo à implementação de terapias mais eficazes em crianças sob tratamento antineoplásico estava na falta de uma análise pontual, válida e viável (Jacobs et al., 2013) ou ausência de instrumentos de fácil manejo, confiáveis e válidos para avaliar a mucosite oral (Tholinson et al., 2009). A continuidade das pesquisas levou à construção da Child's Internacional Mucositis Evaluation Scale, visando suprir a ausência identificada na literatura consultada (Tholinson et al., 2010a, 2020b; Jacobs et al., 2013).

Os relatos sobre o desenvolvimento da Child's Internacional Mucositis Evaluation Scale (ChIMES) foram feitos por Jacobs et al. e por Tholinson et al, esses últimos identificados na fase da pesquisa narrativa. O desenvolvimento dessa escala centrou-se no fato de os pacientes pediátricos não terem condição de cooperar com a avaliação da mucosite em outras escalas por serem longas e de elas não contemplarem questões específicas da mucosite oral (Jacobs et al., 2013). Os resultados mostraram que a metodologia adotada para desenvolvimento da ChIMES foi ampla: revisão de escalas em vigência e consultas à literatura para a manutenção de itens e inserção de novos; instituição de grupo focal com equipe multidisciplinar para avaliação dos itens referentes aos efeitos da mucosite oral sobre as funções, graus de ulceração, frequência cardíaca, saturação e outros (Tomlinson et al., 2009a) e percepção de sintomas pelo paciente pediátrico (Tomlinson et al., 2010b), surgindo aí a primeira versão da escala que recebeu o citado nome ChIMES. Verificou-se a urgência de outra análise dos itens por meio de pesquisa à literatura, feita por um grupo nominal (Tomlinson et al., 2010a), com avaliação, revisão e resumo de critérios das escalas em vigor mais empregadas em relação a alguns pontos (Tomlinson et al.,2010b), posteriormente determinando-se a pontuação dos itens (Jacobs et al., 2013). Os testes de compreensibilidade, de validade e de confiabilidade foram realizados em etapas (Tomlinson et al., 2010a). Especialmente, o teste de validade e de viabilidade da implementação, a capacidade de resposta do paciente pediátrico 
e a validade convergente, incluindo a aceitabilidade do instrumento e o entendimento de seu conteúdo, envolveram pacientes e respectivos pais (Jacobs et al., 2013).

\section{Conclusão}

A pesquisa sobre o estado da arte da avaliação da mucosite oral em crianças com câncer e das respectivas formas de intervenção demonstrou cuidado e precisão no trato da questão, com etapas investigativas envolvendo profissionais especialistas em mucosite oral, pediatras, equipe multidisciplinar e a observação dos próprios pacientes e de seus acompanhantes. Com isso, a visão do problema não só foi ampla como de diversos pontos de vista. Nesse sentido, novos estudos são necessários para investigar a melhor intervenção em crianças com mucosite oral durante o tratamento oncológico.

\section{Referências}

Aggarwal, R., Bansal, D., Naru, J., Salaria, M., Rana, A., Minz, R W., Trehan, A. \& Marwaha, K. (2014). HSV-1 as well as HSV-2 is frequent in oral mucosal lesions of children on chemotherapy. Support Care Cancer, 22, 1773-1779.

Allen, G., Logan, R., \& Gue S. (2020). Oral manifestations of cancer treatment in children: a review of the literature. Clinical Journal of Oncology Nursing, 14 (4), 481-490.

Argelagós, A. P., Cárdenas, A. B. C., \& Blanco, J. R. (2014). Protocolos de atención odontológica a pacientes pediátricos oncológicos. Odontol Pediátr, 22 (2), 153-161.

Botelho, L. L. R., de Almeida Cunha, C. C., \& Macedo, M. (2011). O método da revisão integrativa nos estudos organizacionais. Gestão e sociedade, 5(11), $121-136$

Bourdelin M., Daguindau E., Larosa F., Legrand F., Nerich V. \& Deconinck E., Limat S. (2015). La mucite post-allogreffe de cellules souches he'matopor"e'tiques: facteurs de risque, conse'quences cliniques et pre'vention. Pathologie Biologie, 63, 106-110.

Bulut, H. K., \& Tüfekci, F. G. (2016). Honey prevents oral mocositis in children undergoing chemotherapy: A quasi-experimental study with a control group. Complementary Therapies in Medicine, 29, 132-140.

Castro, J. F. L., Abreu, E. G. F., Correia, A. V. L., Brasil, C. M. V., Perez, D. E. C. \& Pedroso, F. P. R. (2013). Low-level laser in prevention and treatment of oral mucositis in pediatric patients with acute lymphoblastic leukemia. Photomedicine and Laser Surgery, 31 (12), $613-618$.

Devi, K. S. \& Allenidekaniab, A. (2019). The relationship of oral care practice at home with mucositis incidence in children with acute lymphoblastic leukemia. Comprehensive Child and Adolescent Nursing, 42 (S1), 56-64.

Figliolia, S. L. C., Oliveira, D. T., Pereira, M. C., Lauris, J. R. P, Maurício, A. R., Oliveira, D. T. \& Mello de Andrea, M. L.(2008). Oral mucositis in acute lymphoblastic leukaemia: analysis of 169 paediatric patients. Oral Diseases, 14, 761-766.

Garrocho-Rangel, J. A., Herrera-Moncada, M., Márquez-Preciado, R., Tejeda-Nava, F., Ortiz-Zamudio, J. J., \& Pozos-Guillén A. (2018). Oral mucositis in paediatric acute lymphoblastic leukemia patients receiving methotrexate-based chemotherapy: case series. European Journal of Paediatric Dentistry, $9 / 3$.

Hashemi A., Bahrololoumi Z., Khaksar Y., Saffarzadeh N., Neamatzade H. \& Foroughi E. (2015). Mouth-rinses for the prevention of chemotherapy induced oral mucositis in children: a systematic review. Iranian Journal of Pediatric Hematology Oncology, 15 (2).

Jacobs S., Baggott C., Agarwal R., Hesser T., Schechter T., Judd P., Tomlinson D., Beyene J. \& Sung L. (2013). Validation of the Children's International Mucositis Evaluation Scale (ChIMES) in paediatric cancer and SCT. British Journal of Cancer, 109, $2515-2522$.

Kuhn A., Porto F. A., Miraglia P. \& Brunetto A.L. (2009) Low-level infrared laser therapy in chemotherapy-induced oral mucositis. A randomized placebocontrolled trial in children. J Pediatr Hematol Oncol, 31 (1), 33-8.

Mendonça, R. M. H., Araujo, M., Levy, C. E., Morari, J., Silva, R. A. \& Yunes, J. A., \& Brandalise, S. R. (2015). Oral mucositis in pediatric acute lymphoblastic leukemia patients: evaluation of microbiological and hematological factors. Pediatric Hematology and Oncology, 1(9).

Otmani, N., Raouf, A., Hessissen, L. L., Mokhtari, A., Soulaymani, A., \& Khattab, M. (2011). Determinants of severe oral mucositis in paediatric cancer patients: a prospective study. International Journal of Paediatric Dentistry, 21, 210- 216.

Paiva B. S. R., Barroso E. M., Cadamuro S. A., Paula L. A. B., Pirola W. E, Serrano C. V. M. P. \& Paiva C. E. (2018). The Children's International Mucositis Evaluation Scale is valid and reliable for the assessment of mucositis among brazilian children with cancer. Journal of Pain and Sympton Management, 56(5), 774-783.

Schubert M. M., Williams B. E., Lloid M. E., Donaldson G. \& Chapko M. K. (1992). Clinical assessment scale for the rating of oral mucosal changes associated with bone marrow transplantation: development of an oral mucositis index. Cancer, 69, 2469-77. Recuperado em 1 fevereiro 2021 , de https://acsjournals.online library.wiley.com/doi/pdfdirect/10.1002/1097-0142(19920515)69.

Singh R., Sharma S., Kaur S., Medhi B., Trehan A. \& Bijarania S. K. (2019) Effectiveness of topical application of honey on oral mucosa of children for the management of oral mucositis associated with chemotherapy. The Indian Journal of Pediatrics, 86(3), 224-228. 
Research, Society and Development, v. 10, n. 11, e435101120018, 2021

(CC BY 4.0) | ISSN 2525-3409 | DOI: http://dx.doi.org/10.33448/rsd-v10i11.20018

Sonis S. T., Eilers J. P., Epstein J. B., Le Veque F. G., Liggett Jr W. H . \& Mulagha M. T.(1999) Validation of a new scoring system for the assessment of clinical trial research of oral mucositis induced by radiation or chemotherapy. Am Cancer Soc. 85, 2103-13.

Stiff P. J., Erder H., Bensinger W. I., Emmanouilides C., Gentile T., Isitt J., Lu Z. J. \& Spielberger R. (2006) Reliability and validity of a patient self-administered daily questionnaire to assess impact of oral mucositis (OM) on pain and daily functioning in patients undergoing autologous hematopoietic stem cell transplantation (HSCT). Bone Marrow Transplantation, 37(4), 393-401.

Sung L., Robinson P., Treister N., Baggott T., Gibson P., Tissing W., Wiernikowski J. \& Brinklow J, Dupuis L. L. (2017) Guideline for the prevention of oral and oropharyngeal mucositis in children receiving treatment for cancer or undergoing haematopoietic stem cell transplantation. Supportive \& Palliative Care, 7, 7-16.

Tomlinson D., Gibson F., Treister N., Baggott C., Judd J. P., Hendershot E., Maloney A. M., Doyle J., Feldman B., Kwong K. \& Sung L. (2009a) Understandability, content validity, and overall acceptability of the Children's International Mucositis Evaluation Scale (ChIMES) Child and parent reporting. J Pediatr Hematol Oncol, 31 (6), 416-24.

Tomlinson D., Gibson F., Treister F., Baggott C., Judd P., Hendershot E., Maloney A. M., Doyle J., Feldman B., Kwong K. \& Sung L. (2010a) Refinement of the Children's International Mucositis Evaluation Scale (ChIMES): child and parent perspectives on understandability, content validity and acceptability. European Journal of Oncology Nursing, 14, 29-41.

Tomlinson D., von Baeyer C. L., Stinso J. N. \& Sung L. (2010b) A systematic review of faces scales for the self-report of pain intensity in children. Pediatrics, 126(5), 1168-98. https:// pubmed.ncbi.nlm.nih.gov/20921070/

WHO World Health Organization. (1979) WHO handbook for reporting results of cancer treatment. https://apps.who.int/iris/handle/10665/37200. 